\title{
Reaching the Chu Lower Bound on Q With Magnetic Dipole Antennas Using a Magnetic-Coated PEC Core
}

\section{Kim, Oleksiy S.; Breinbjerg, Olav}

Published in:

I E E E Transactions on Antennas and Propagation

Link to article, DOI:

10.1109/TAP.2011.2158965

Publication date:

2011

Document Version

Early version, also known as pre-print

Link back to DTU Orbit

Citation (APA):

Kim, O. S., \& Breinbjerg, O. (2011). Reaching the Chu Lower Bound on Q With Magnetic Dipole Antennas Using a Magnetic-Coated PEC Core. I E E E Transactions on Antennas and Propagation, 59(8), 2799-2805. https://doi.org/10.1109/TAP.2011.2158965

\section{General rights}

Copyright and moral rights for the publications made accessible in the public portal are retained by the authors and/or other copyright owners and it is a condition of accessing publications that users recognise and abide by the legal requirements associated with these rights.

- Users may download and print one copy of any publication from the public portal for the purpose of private study or research.

- You may not further distribute the material or use it for any profit-making activity or commercial gain

- You may freely distribute the URL identifying the publication in the public portal 


\title{
Reaching the Chu lower bound on Q with magnetic dipole antennas using a magnetic-coated PEC core
}

\author{
Oleksiy S. Kim and Olav Breinbjerg, Member, IEEE
}

\begin{abstract}
We analytically solve the radiation problem for a spherical magnetic dipole antenna with a material-coated perfectly electrically conducting core. Using the closed-form expressions derived for the internal and external stored energies as well as for the radiation quality factor $Q$, we determine the optimal geometrical and material parameters of the antenna. We show that the optimal permeability of the coating increases as the coating becomes thinner; as a result, the energy stored in it and the radiation quality factor $Q$ reduce. In the limit of an infinitely thin coating, the optimal permeability tends to infinity, the internal stored energy vanishes, and the $Q$ reaches the Chu lower bound, irrespective of the antenna electrical size $k a$ and permittivity of the coating.
\end{abstract}

Index Terms-Electrically small antennas, fundamental limitations, magnetic dipole, quality factor, Chu lower bound

\section{INTRODUCTION}

$\mathbf{I}$ $\mathrm{N}$ his seminal work from 1948 [1] Chu established a lower bound for the radiation quality factor $Q$ of an electrically small antenna; this can be expressed as [1]-[3]

$$
Q_{\mathrm{Chu}}=\frac{1}{(k a)^{3}}+\frac{1}{k a}
$$

with $k$ being the free-space wave number and $a$ the radius of the smallest sphere circumscribing the antenna. The Chu lower bound is generally considered to be too optimistic, since it takes into account only the stored energy in the region external to the circumscribing sphere. If the stored energy in the internal region is also taken into account, the $Q$ will increase. For a spherical electric surface current distribution with free-space internal region - a so-called air-core - it has been shown [4]-[6] that the quality factor $Q$ is limited from above to $1.5 Q_{\mathrm{Chu}}$ for electric dipole antennas (radiating the $\mathrm{TM}_{10}$ spherical mode) and $3.0 Q_{\mathrm{Chu}}$ for magnetic dipole antennas (radiating the $\mathrm{TE}_{10}$ spherical mode) as $k a \rightarrow 0$. A range of practical antennas [7]-[12] confirm these values.

There is obviously a strong interest in antennas that overcome the limits of the air-core spherical antennas and approach the Chu lower bound. The lower $Q$ requires the internal stored energy - electric energy for electric dipole antennas and magnetic energy for magnetic dipole antennas - to be reduced. One way to do it was pointed out by Wheeler in his work from 1958 [4]. Wheeler showed that the Chu lower bound can be reached by a magnetic dipole antenna with a solid spherical magnetic core of infinite permeability - but only in the limit

O. S. Kim and O. Breinbjerg are with the Department of Electrical Engineering, Electromagnetic Systems, Technical University of Denmark, DK-2800 Kgs. Lyngby, Denmark (e-mail: \{osk,ob\}@elektro.dtu.dk) $k a \rightarrow 0$. For a finite-size magnetic dipole antenna the quality factor is proved to be [13], [14]

$$
Q \approx\left\{1+0.366(k a)^{2}\right\} Q_{\mathrm{Chu}}
$$

On one hand, the expression (2) suggests that the $Q$ can approach the Chu lower bound $Q_{\mathrm{Chu}}$ very closely; e.g., within $10 \%$ for $k a=0.5$. On the other hand, it excludes that $Q_{\mathrm{Chu}}$ can be reached by a finite-size magnetic dipole antenna with a solid spherical magnetic core. The limiting mechanism is the internal resonances, which start occurring when the electrical size of the core reaches specific values. However, they may be avoided if a metal sphere is introduced into a solid magnetic core, as shown in this paper.

Another way to lower the $Q$ follows from the Huygens-Love principle, which states that equivalent electric and magnetic currents on a surface enclosing a source radiate the same fields as the original source. Furthermore, the equivalent currents produce zero fields internally to the surface they reside on. Thus, equivalent electric and magnetic surface currents being a source itself represent an ideal antenna with no internal stored energy and the lowest possible $Q$ [15], [16]. The principle is used in [17], [18] to derive lower bounds on the $Q$ for electrically small antennas of arbitrary shape. In subsequent works by the same authors [19], [20], it is suggested to utilize a thin sheet of high-permeable magnetic material to produce magnetic polarization currents as an alternative to the current of magnetic charges. Indeed, a numerical investigation of an electric dipole antenna [19], [20] illustrates that the air-core limit can be overcome and the Chu lower bound $Q_{\text {Chu }}$ closely approached.

The purpose of this paper is to show that the Chu lower bound can be approached even closer than (2) for a finitesize magnetic dipole antenna using a material-coated perfectly electrically conducting (PEC) core. This facilitates electrically small antenna designs of the best possible performace with respect to the quality factor and thus bandwidth. Preliminary results of this work were presented in [21], and similar results were independently reported in [22]. Here, we present the details of the analytical solution for the problem (Section II) and determine the optimal parameters of the antenna ensuring the lowest possible ratio $Q / Q_{\text {Chu }}$ (Section III).

The time factor $\exp (-i \omega t)$ is assumed and suppressed throughout the paper.

\section{THEORY AND RESUlTS}

The geometry of the antenna is sketched in Fig. 1. A spherical PEC core of radius $b$ is coated with a concentric 


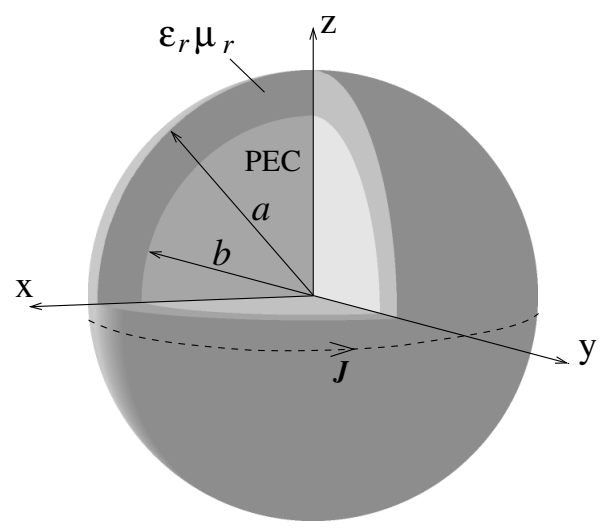

Fig. 1. Magnetic dipole antenna with a material-coated PEC core. Timeharmonic surface current density $\mathbf{J}$ is distributed over the surface of the antenna.

magnetodielectric shell of external radius $a$. The material of the shell is linear, isotropic, homogeneous, lossless and dispersion-less with the relative permittivity $\varepsilon_{\mathrm{r}}$, relative permeability $\mu_{\mathrm{r}}$, and wave number $k_{s}=\sqrt{\varepsilon_{\mathrm{r}} \mu_{\mathrm{r}}} k$. In a spherical coordinate system $(r \theta \phi)$ with the origin at the center of the core, an impressed electric current density on the surface of the shell is defined as

$$
\mathbf{J}=\hat{\mathbf{a}}_{\phi} J_{0} \sin \theta
$$

where $\hat{\mathbf{a}}_{\phi}$ is the azimuthal unit vector and $J_{0}$ is the amplitude $(\mathrm{A} / \mathrm{m})$. In the free space, externally to the antenna, this current radiates electromagnetic fields equal to those of a $z$-directed elementary magnetic dipole located at the origin.

\section{A. Analytical Solution}

The electric and magnetic fields in the material shell, $\mathbf{E}^{-}(\mathbf{r})$ and $\mathbf{H}^{-}(\mathbf{r})$, and in free space, $\mathbf{E}^{+}(\mathbf{r})$ and $\mathbf{H}^{+}(\mathbf{r})$, are expressed in terms of vector spherical wave functions as

$$
\begin{aligned}
& \mathbf{E}^{-}(\mathbf{r})=\frac{k_{s}}{\sqrt{\eta_{s}}} C^{-} \mathbf{F}_{101}^{(5)}(\mathbf{r}) \\
& \mathbf{H}^{-}(\mathbf{r})=-i k_{s} \sqrt{\eta_{s}} C^{-} \mathbf{F}_{201}^{(5)}(\mathbf{r}) \\
& \mathbf{E}^{+}(\mathbf{r})=\frac{k}{\sqrt{\eta}} C^{+} \mathbf{F}_{101}^{(3)}(\mathbf{r}) \\
& \mathbf{H}^{+}(\mathbf{r})=-i k \sqrt{\eta} C^{+} \mathbf{F}_{201}^{(3)}(\mathbf{r})
\end{aligned}
$$

where $\eta_{s}$ and $\eta$ are the intrinsic admittances of the shell and free space, respectively, and $C^{ \pm}$are coefficients to be determined. The $\mathbf{F}$-functions are defined using the notation of
Hansen [23] as

$$
\begin{aligned}
\mathbf{F}_{101}^{(5)}(\mathbf{r})= & \frac{\sqrt{6}}{4 \sqrt{\pi}} z_{1}^{(5)}\left(k_{s} r\right) \sin \theta \hat{\mathbf{a}}_{\phi} \\
\mathbf{F}_{201}^{(5)}(\mathbf{r})= & \frac{\sqrt{6}}{2 \sqrt{\pi}} \frac{z_{1}^{(5)}\left(k_{s} r\right)}{k_{s} r} \cos \theta \hat{\mathbf{a}}_{r} \\
& -\frac{\sqrt{6}}{4 \sqrt{\pi}} \frac{1}{k_{s} r} \frac{d}{d\left(k_{s} r\right)}\left\{k_{s} r z_{1}^{(5)}\left(k_{s} r\right)\right\} \sin \theta \hat{\mathbf{a}}_{\theta} \\
\mathbf{F}_{101}^{(3)}(\mathbf{r})= & \frac{\sqrt{6}}{4 \sqrt{\pi}} z_{1}^{(3)}(k r) \sin \theta \hat{\mathbf{a}}_{\phi} \\
\mathbf{F}_{201}^{(3)}(\mathbf{r})= & \frac{\sqrt{6}}{2 \sqrt{\pi}} \frac{z_{1}^{(3)}(k r)}{k r} \cos \theta \hat{\mathbf{a}}_{r} \\
& -\frac{\sqrt{6}}{4 \sqrt{\pi}} \frac{1}{k r} \frac{d}{d(k r)}\left\{k r z_{1}^{(3)}(k r)\right\} \sin \theta \hat{\mathbf{a}}_{\theta} .
\end{aligned}
$$

The radial dependence of the fields in free space is represented by the spherical Hankel function of the first kind $z_{1}^{(3)}(k r)=h_{1}^{(1)}(k r)$, whereas for the shell an appropriate linear combination of spherical Bessel and Neumann functions $z_{1}^{(5)}\left(k_{s} r\right)=j_{1}\left(k_{s} b\right) y_{1}\left(k_{s} r\right)-y_{1}\left(k_{s} b\right) j_{1}\left(k_{s} r\right)$ is introduced, so that the boundary condition at the surface of the PEC core is satisfied.

By substituting (5) into (4) and enforcing the boundary conditions at the surface of the antenna $r=a$, the coefficients $C^{ \pm}$can be determined in closed form as

$$
\begin{aligned}
& C^{-}=J_{0} i a \frac{\sqrt{\eta_{s}}}{\eta} \frac{4 \sqrt{\pi}}{\sqrt{6}} k a h_{1}^{(1)}(k a) I^{-1} \\
& C^{+}=J_{0} i a \frac{1}{\sqrt{\eta}} \frac{4 \sqrt{\pi}}{\sqrt{6}} k_{s} a z_{1}^{(5)}\left(k_{s} a\right) I^{-1}
\end{aligned}
$$

where

$$
\begin{aligned}
I= & \frac{\eta_{s}}{\eta} k a h_{1}^{(1)}(k a) \frac{d}{d\left(k_{s} a\right)}\left\{k_{s} a z_{1}^{(5)}\left(k_{s} a\right)\right\} \\
& -k_{s} a z_{1}^{(5)}\left(k_{s} a\right) \frac{d}{d(k a)}\left\{k a h_{1}^{(1)}(k a)\right\} .
\end{aligned}
$$

\section{B. Stored Energy and Quality Factor}

To determine the stored energies, the fields in (4) are spatially integrated over the respective regions. In free space, the contribution of the propagating field is subtracted, so that only the energy density of the non-propagating field remains [2]. The resulting expressions for the internal electric $W_{\mathrm{E}}^{-}$, internal magnetic $W_{\mathrm{H}}^{-}$, external electric $W_{\mathrm{E}}^{+}$, and external magnetic $W_{\mathrm{H}}^{+}$stored energy are found to be

$$
\begin{aligned}
W_{\mathrm{E}}^{-}= & \frac{1}{4 \omega}\left|C^{-}\right|^{2}\left\{\frac{\left(k_{s} a\right)^{3}}{2}\left[R_{1}^{2}\left(k_{s} a\right)-R_{0}\left(k_{s} a\right) R_{2}\left(k_{s} a\right)\right]\right. \\
& \left.+\frac{\left(k_{s} b\right)^{3}}{2} R_{0}\left(k_{s} b\right) R_{2}\left(k_{s} b\right)\right\} \\
W_{\mathrm{H}}^{-}= & \frac{1}{4 \omega}\left|C^{-}\right|^{2}\left\{\frac{\left(k_{s} a\right)^{3}}{2}\left[R_{0}^{2}\left(k_{s} a\right)-R_{-1}\left(k_{s} a\right) R_{1}\left(k_{s} a\right)\right]\right. \\
W_{\mathrm{E}}^{+}= & \frac{1}{4 \omega}\left|C^{+}\right|^{2} \frac{1}{k a} \\
W_{\mathrm{H}}^{+}= & \frac{1}{4 \omega}\left|C^{+}\right|^{2}\left\{\frac{1}{(k a)^{3}}+\frac{1}{k a}\right\}
\end{aligned}
$$


where $R_{n}\left(k_{s} r\right)=j_{1}\left(k_{s} b\right) y_{n}\left(k_{s} r\right)-y_{1}\left(k_{s} b\right) j_{n}\left(k_{s} r\right)$. The expressions for the external stored energies, $(8 \mathrm{c})$ and $(8 \mathrm{~d})$, have been reported previously [2], [13], [24], whereas the expressions for the stored energies in the spherical shell enclosing the PEC sphere, (8a) and (8b), are new.

The radiation quality factor $Q$ is determined from

$$
Q=2 \omega \frac{\max \left(W_{\mathrm{H}}^{-}+W_{\mathrm{H}}^{+}, W_{\mathrm{E}}^{-}+W_{\mathrm{E}}^{+}\right)}{P_{\mathrm{rad}}}
$$

using (6), (8), and the radiated power

$$
P_{\text {rad }}=\frac{1}{2}\left|C^{+}\right|^{2} .
$$

Expression (9) assumes that a resonant system is established by increasing the lesser of the electric and magnetic energies to equal the larger through use of, e.g., a lumped-component tuning circuit.

\section{Results}

The effect of the magnetic-coated PEC core $\left(\varepsilon_{\mathrm{r}}=1\right)$ is illustrated in Fig. 2. In Fig. 2a, the ratio of the total stored electric energy $W_{\mathrm{E}}=W_{\mathrm{E}}^{+}+W_{\mathrm{E}}^{-}$to the total stored magnetic energy $W_{\mathrm{H}}=W_{\mathrm{H}}^{+}+W_{\mathrm{H}}^{-}$is plotted vs. the relative permeability $\mu_{\mathrm{r}}$ of the shell for three values of the relative radius of the PEC core $b / a=0.0,0.65$, and 0.9 with fixed external electrical radius $k a=0.5$. For the permeability close to the free space value, the magnetic energy clearly dominates irrespective of $b / a$. As the permeability increases, the relative part of the stored electric energy becomes larger, and eventually the first resonance is reached.

Figure $2 \mathrm{~b}$ shows the ratio of the internal stored energy $W^{-}=W_{\mathrm{E}}^{-}+W_{\mathrm{H}}^{-}$to the total stored energy $W_{\text {Total }}=W_{\mathrm{E}}^{-}+W_{\mathrm{H}}^{-}+W_{\mathrm{E}}^{+}+W_{\mathrm{H}}^{+}$vs. the relative permeability $\mu_{\mathrm{r}}$. The internal stored energy, which is predominantly magnetic, initially decreases with $\mu_{\mathrm{r}}$; however, as $\mu_{\mathrm{r}}$ passes an optimum value, it starts rising towards the internal resonance, at which the total stored energy is entirely internal.

The radiation quality factor $Q$ demonstrates similar behaviour, as seen in Fig. 2c, where the $Q$, normalized by the Chu lower bound $Q_{\text {Chu }}$, is plotted vs. the relative permeability $\mu_{\mathrm{r}}$. The ratio $Q / Q_{\text {Chu }}$ is lowest for some optimal $\mu_{\mathrm{r}}$, and it becomes of course infinite at the internal resonances.

In the free space case $\left(\mu_{\mathrm{r}}=1\right)$, the ratio $W^{-} / W_{\text {Total }}$ increases as the PEC core becomes relatively larger (Fig. 2b). The source currents on the surface of the antenna induce oppositely directed currents on the surface of the PEC core, and as the PEC surface becomes closer to the source currents, the external fields - and thus the external stored energy and the radiated power - reduce. In the limiting case $b / a \rightarrow 1$ and $\mu_{\mathrm{r}}=1$, the ratio $W^{-} / W_{\text {Total }}$ tends to unity, and the $Q$ tends to infinity.

The most prominent effect of the magnetic-coated PEC core is that the larger $b / a$, the higher the value of the permeability $\mu_{\mathrm{r}}$ at which the first resonance occurs. The optimal value of the permeability, at which the ratios $W^{-} / W_{\text {Total }}$ and $Q / Q_{\text {Chu }}$ are minimal, becomes correspondingly higher. Potentially, an arbitrary high $\mu_{\mathrm{r}}$ can be used - and an arbitrary low ratio $W^{-} / W_{\text {Total }}$ achieved - without the risk of hitting a resonance

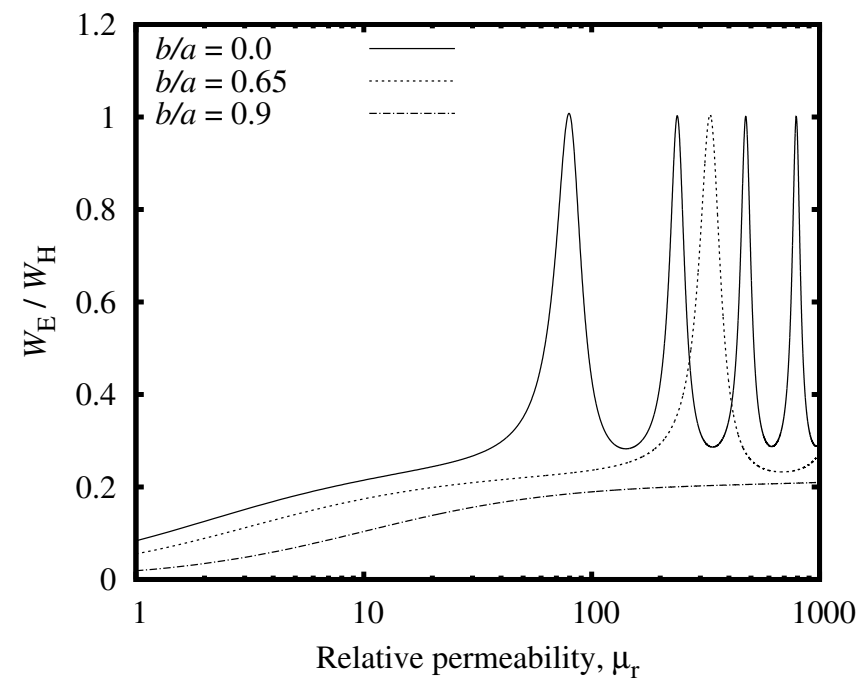

(a)

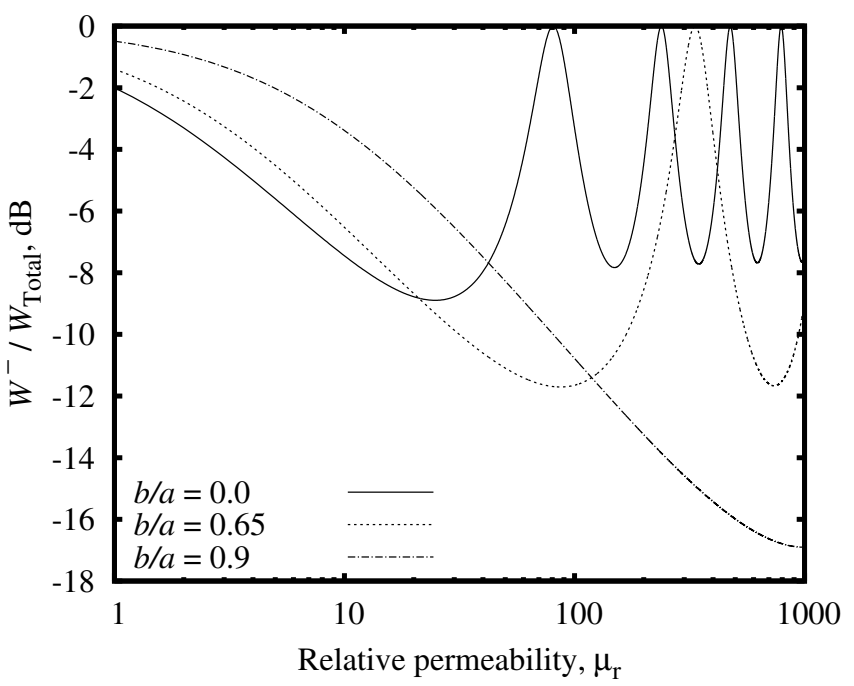

(b)

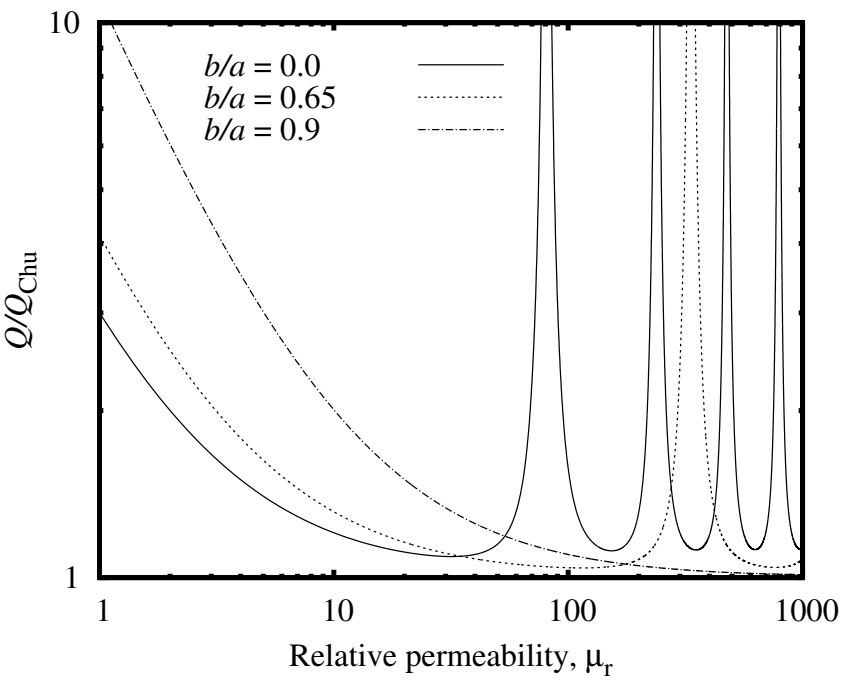

(c)

Fig. 2. Energy and quality factor as functions of $\mu_{\mathrm{r}}\left(\varepsilon_{\mathrm{r}}=1\right)$ for fixed $k a=0.5$ : (a) Ratio of electric and magnetic stored energies; (b) Ratio of internal and total stored energy; (c) Quality factor (normalized by Chu lower bound). 
if the shell is thin enough. In the limit of an infinitely thin magnetic shell $(b / a \rightarrow 1)$, the optimal $\mu_{\mathrm{r}}$ is infinitely high, the magnetic energy stored in the shell vanishes, and the $Q$ reaches the Chu lower bound $Q_{\text {Chu }}$.

\section{OPTIMAL PARAMETERS}

In this section, we determine the optimal geometrical and material parameters minimizing the ratio $Q / Q_{\text {Chu }}$ for a spherical magnetic dipole antenna with material-coated PEC core. First, the optimal parameters are found from the exact analytical solution presented in Section II. Second, a simple standing wave model that provides a physical insight into the problem of determining the optimal parameters is discussed.

\section{A. Optimal Parameters Extracted from the Exact Solution}

Assuming that all parameters are chosen such that the stored magnetic energy exceeds the stored electric energy, $W_{\mathrm{H}}>W_{\mathrm{E}}$, the expression for the radiation quality factor $Q$ can be written from (6)-(10) as

$$
Q=2 \omega \frac{W_{\mathrm{H}}^{-}+W_{\mathrm{H}}^{+}}{P_{\mathrm{rad}}}=\left\{1+\frac{1}{\mu_{\mathrm{r}}} \Psi\left(k_{s} a, b / a\right)\right\} Q_{\mathrm{Chu}}
$$

where

$$
\begin{aligned}
\Psi\left(k_{s} a, b / a\right)= & \frac{\left(k_{s} a\right)^{2}}{2 R_{1}^{2}\left(k_{s} a\right)}\left\{R_{0}^{2}\left(k_{s} a\right)-(b / a)^{3} R_{0}^{2}\left(k_{s} b\right)\right. \\
& \left.-R_{-1}\left(k_{s} a\right) R_{1}\left(k_{s} a\right)\right\}-1 .
\end{aligned}
$$

1) Optimal $\mu_{r}$ for given $k a, \varepsilon_{r}$ and $b / a$ : The minimum of $Q$ in (11) with respect to the relative permeability $\mu_{\mathrm{r}}$ of the shell is found by solving the equation

$$
\frac{\partial Q}{\partial \mu_{\mathrm{r}}}=\frac{\left(k_{s} a\right)}{2} \frac{\partial \Psi\left(k_{s} a, b / a\right)}{\partial\left(k_{s} a\right)}-\Psi\left(k_{s} a\right)=0 .
$$

Since $\Psi\left(k_{s} a, b / a\right)$ in (12) is an oscillating function, equation (13) has infinitely many solutions - values of $k_{s} a$ - for any value of $b / a$. Denoting these solutions as $\Phi_{n}(b / a)$, the optimal permeability of the shell for a given $k a$ and $\varepsilon_{\mathrm{r}}$ is determined as

$$
\mu_{\mathrm{r}}^{\mathrm{opt}}=\frac{\Phi_{n}^{2}(b / a)}{\varepsilon_{\mathrm{r}}(k a)^{2}}
$$

Substituting (14) into (11), we obtain the lowest achievable $Q$ for each $n$ as

$$
Q=\left\{1+\varepsilon_{\mathrm{r}}(k a)^{2} \frac{\Psi\left(\Phi_{n}(b / a), b / a\right)}{\Phi_{n}^{2}(b / a)}\right\} Q_{\mathrm{Chu}} .
$$

The functions $\Phi_{n}(b / a)$ are plotted in Fig. 3a for three values of $n=1,2$, and 3 ; the corresponding ratios $Q / Q_{\text {Chu }}$ are shown in Fig. $3 \mathrm{~b}$ for $k a \sqrt{\varepsilon_{\mathrm{r}}}=1.0$. It is observed that the solutions with higher $n$ yield higher ratios $Q / Q_{\mathrm{Chu}}$, and though the difference becomes negligible as $b / a \rightarrow 1$, the first solution $n=1$ is of most interest. As evident from (15), this observation holds for arbitrary $k a \sqrt{\varepsilon_{\mathrm{r}}}$.

Use of plotted data is not always convenient. Therefore, we applied a least-squares curve fitting of a rational function

$$
\Phi_{1}(x) \approx u_{1} \frac{1+A x+B x^{2}}{1-x^{2}}
$$

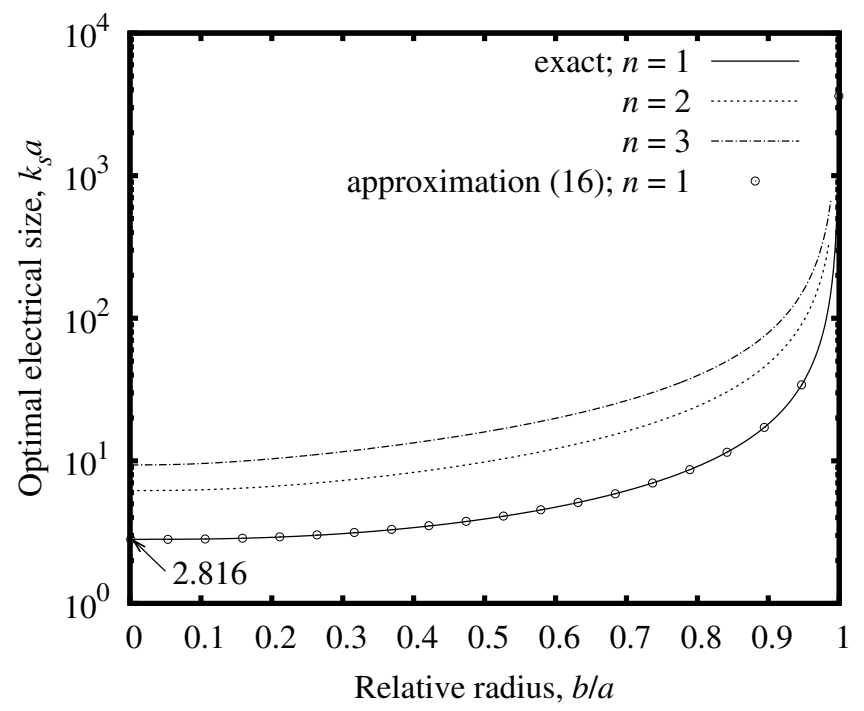

(a)

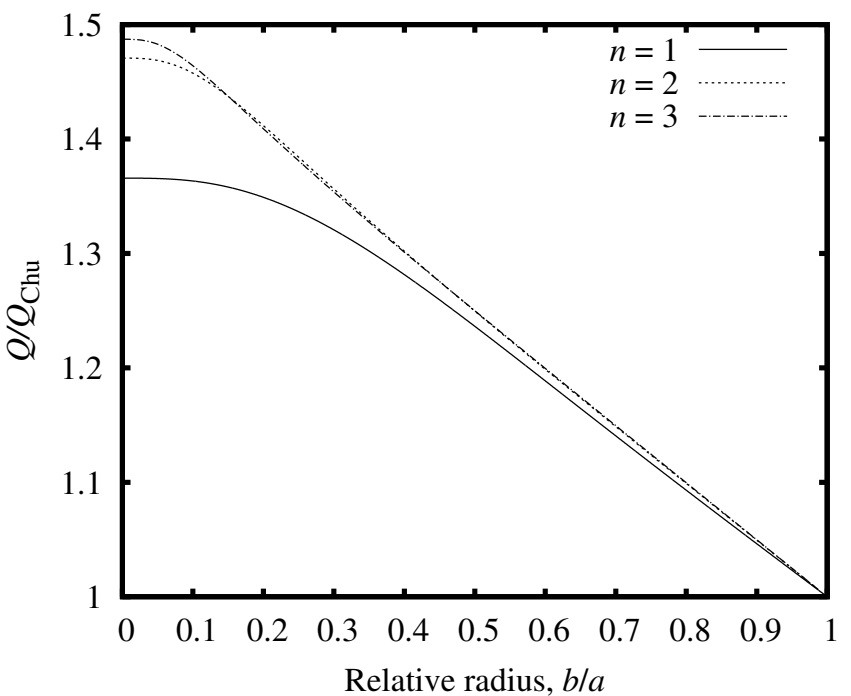

(b)

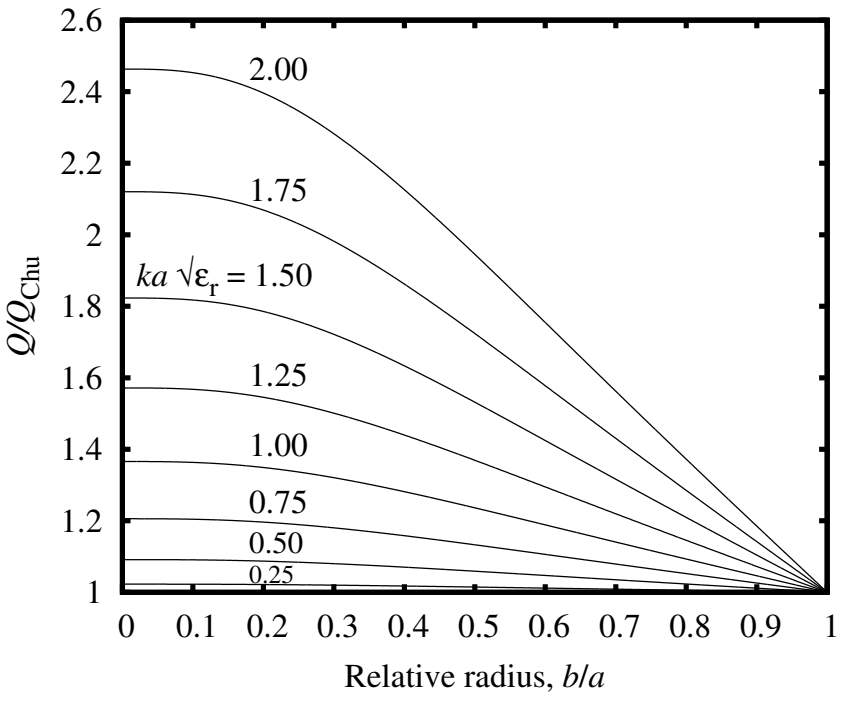

(c)

Fig. 3. Optimal electrical size $k_{s} a$ (a) and the corresponding lowest achievable ratio $Q / Q_{\text {Chu }}$ : (b) for $n=1,2,3$, and $k a \sqrt{\varepsilon_{\mathrm{r}}}=1.0$; (c) for various $k a \sqrt{\varepsilon_{\mathrm{r}}}$ and $n=1$. 
to the numerically found data points of $\Phi_{1}(b / a)$. In (16), $u_{1} \approx 2.8161$ is a constant determined in [14] while solving a problem for a spherical magnetic dipole antenna with a solid material core $(b / a=0)$. Constants $A \approx-0.1048$ and $B \approx 0.3894$ are found to yield a root mean square error of $1.4 \%$; the resulting approximation is compared to the exact curve in Fig. 3a.

The dependence of the lowest achievable ratio $Q / Q_{\text {Chu }}$ vs. the relative radius of the PEC core $b / a$ is presented in Fig. 3c for various values of $k a \sqrt{\varepsilon_{\mathrm{r}}}$. The plot is based on (15) with $n=1$. It is observed, that irrespective of the antenna electrical size, the Chu lower bound $Q_{\text {Chu }}$ can be reached as the shell becomes infinitely thin $(b / a \rightarrow 1)$. For other shell thicknesses, the lowest achievable ratio $Q / Q_{\text {Chu }}$ depends on $k a \sqrt{\varepsilon_{\mathrm{r}}}$. Particularly, for a fixed $k a$, the $Q$ deteriorates as the relative permittivity $\varepsilon_{\mathrm{r}}$ of the shell increases. The similar dependence was observed for a magnetic dipole antenna with a solid magnetodielectric core [13], [14]. However, with a materialcoated PEC core the deteriorating effect of the permittivity can be mitigated by decreasing the shell thickness (increasing $b / a)$. For instance, an antenna with $k a \sqrt{\varepsilon_{\mathrm{r}}}=k a=0.5$ and $b / a=0.135$ yields $Q / Q_{\text {Chu }}=1.09$. Changing permittivity from 1 to 16 results in $k a \sqrt{\varepsilon_{\mathrm{r}}}=2.0$ and brings $Q / Q_{\text {Chu }}$ up to 2.44 . By making the shell thinner, so that $b / a=0.952$, the ratio $Q / Q_{\text {Chu }}$ can be reduced to its initial value 1.09. Although, in all cases the relative permittivity of the shell $\mu_{\mathrm{r}}$ is assumed to be optimal according to (14), this prerequisite is not very strict, due to the wide minimum seen in Fig. 2c.

If the electrical size of the antenna $k a$, the radius of the core $b$ and the permittivity of the coating $\varepsilon_{\mathrm{r}}$ are fixed, the optimum size $k_{s} a$ is found from either (16) or Fig. 3a. The optimum permeability of the coating $\mu_{\mathrm{r}}$ is then determined using (14), and the lowest achievable $Q$ is given by (15) or Fig. 3c.

2) Optimal b/a for given $k a, \mu_{r}$ and $\varepsilon_{r}$ : As shown above, the advantage of a material-coated PEC core versus a solid material core is that a $Q$ very close to the Chu lower bound can be achieved for a finite-size magnetic dipole antenna. This is done using a very thin magnetic coating of a high-permeable material with no restrictions on its permittivity. However, a free choice of material permeability is not usually possible in practice. Therefore, it is useful determine optimal relative radius of the PEC core $b / a$ for selected antenna electrical size $k a$, and coating permeability $\mu_{\mathrm{r}}$ and permittivity $\varepsilon_{\mathrm{r}}$. The problem reduces to solving the following equation

$$
\frac{\partial Q}{\partial(b / a)}=\frac{\partial \Psi\left(k_{s} a, b / a\right)}{\partial(b / a)}=0
$$

Similar to (13), the equation (17) can have multiple solutions as

$$
b / a=F_{n}\left(k_{s} a\right)
$$

The first three ones are shown in Fig. 4a. Note, that $F_{n}\left(k_{s} a\right)$ is not the inverse of the $\Phi_{n}(b / a)$ function. For instance, for $n=1$, the optimal $k_{s} a$ for a solid core $(b / a=0)$ is $k_{s} a=$ $u_{1} \approx 2.816$ (Fig. 3a), whereas the value of $k_{s} a$ for which the optimal $b / a=0$ is approximately 2.083 (Fig. 4a).

Substituting (18) into (11) yields the lowest achievable $Q$

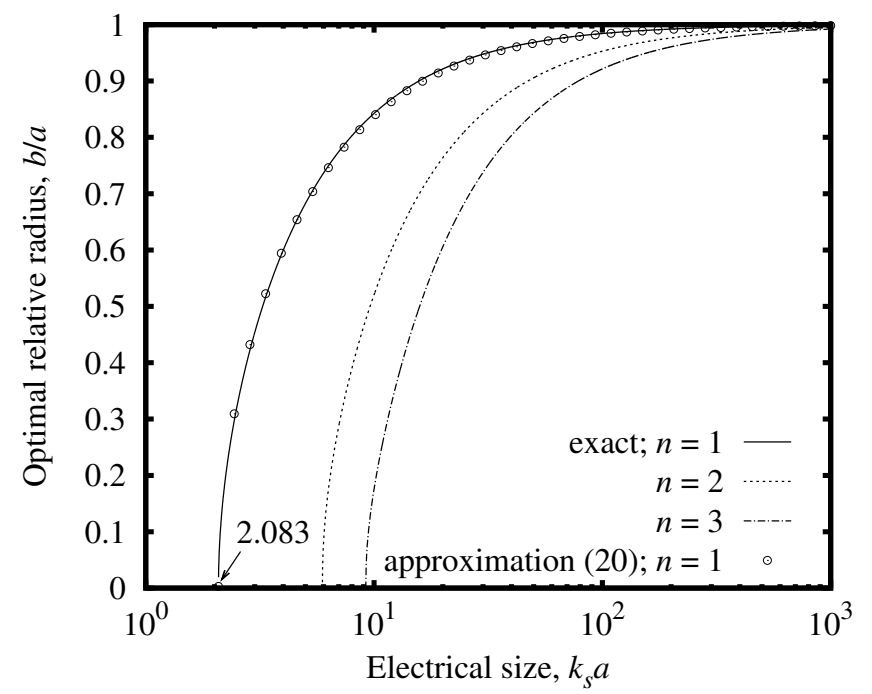

(a)

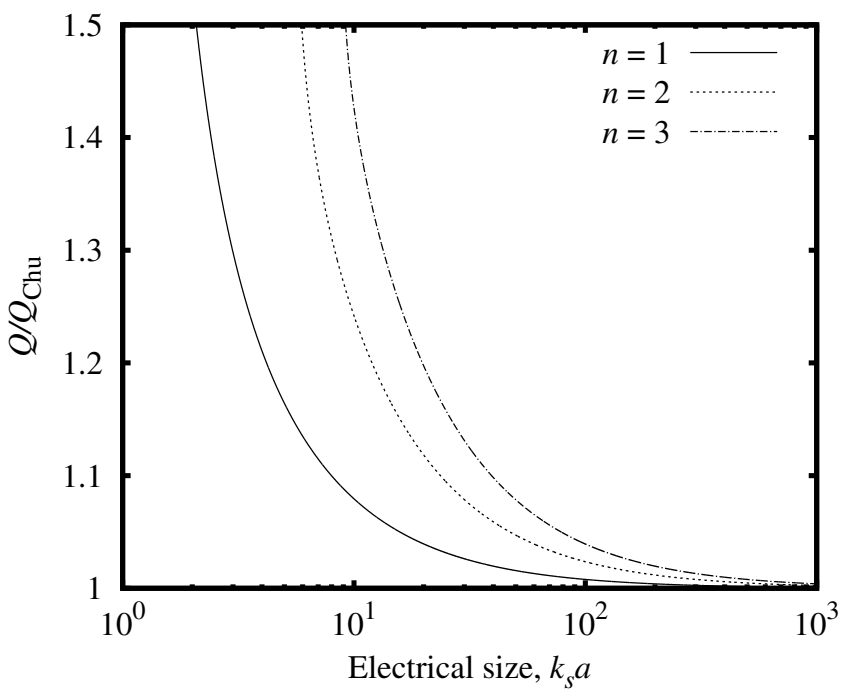

(b)

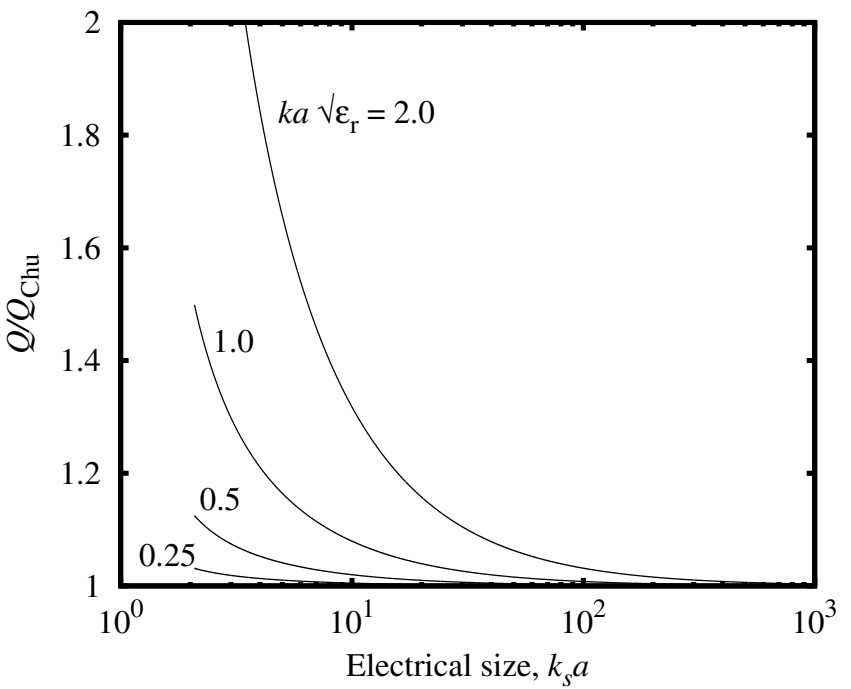

(c)

Fig. 4. Optimal relative radius of the PEC core $b / a$ (a) and the corresponding lowest achievable ratio $Q / Q_{\text {Chu }}$ : (b) for $n=1,2,3$, and $k a \sqrt{\varepsilon_{\mathrm{r}}}=1.0$; (c) for various $k a \sqrt{\varepsilon_{\mathrm{r}}}$ and $n=1$. 
for given $k_{s} a$ and $n$

$$
Q=\left\{1+\varepsilon_{\mathrm{r}}(k a)^{2} \frac{\Psi\left(k_{s} a, F_{n}\left(k_{s} a\right)\right)}{\left(k_{s} a\right)^{2}}\right\} Q_{\mathrm{Chu}} .
$$

Again, as in the previous case (Section III-A1), the first solution $n=1$ yields the lowest ratio $Q / Q_{\text {Chu }}$ (Fig. 4b). And as in the previous case, higher values of $k a \sqrt{\varepsilon_{\mathrm{r}}}$ require higher $\mu_{\mathrm{r}}$, and thus higher $k_{s} a$ and $b / a$, to achieve the same ratio $Q / Q_{\text {Chu }}$ (Fig. 4c).

A convenient approximation of $F_{1}\left(k_{s} a\right)$ is found to be

$$
F_{1}(x) \approx \frac{\sqrt{x^{2}-2.083^{2}}}{x+1.671}, x \geq 2.083
$$

which ensures the root mean square error of $0.4 \%$; the fitting is compared to the exact solution in Fig. 4a.

The design of a magnetic dipole antenna with a materialcoated PEC core starts with the antenna electrical size $k a$ and parameters $\mu_{\mathrm{r}}, \varepsilon_{\mathrm{r}}$ of the material. The optimal ratio $b / a$ is determined using Fig. 4a or the approximation (20). The lowest achievable $Q$ is then found using (19) or Fig. 4c for the corresponding $k a \sqrt{\varepsilon_{\mathrm{r}}}$. If $k_{s} a<2.083$, a solid material core should be applied, and the lowest $Q$ is then given by (2).

3) Range of validity: The expressions (11)-(20) are valid as long as $W_{\mathrm{H}}>W_{\mathrm{E}}$, that is, until the first resonance is reached. Figure 5 shows the optimal parameters compared to the parameters for the first resonance found numerically for several values of $k a$ and $\varepsilon_{\mathrm{r}}$. Note, that the results are presented as $k_{s}(a-b) /(2 \pi)=(a-b) / \lambda_{s}$ vs. the relative radius $b / a$, that is, the vertical axis of the plot represents the shell thickness measured in wavelength in the coating material $\lambda_{s}=(2 \pi) / k_{s}$.

To show the optimal parameters found in Sections III-A1 and III-A2 on the same plot in Fig. 5, the expression (14) is transformed as

$$
\frac{a-b}{\lambda_{s}}=\frac{1}{2 \pi}\left(\Phi_{1}(b / a)-(b / a) \Phi_{1}(b / a)\right)
$$

and the expression (18) is casted in a parametric form as

$$
\left\{\begin{aligned}
\frac{a-b}{\lambda_{s}} & =\frac{a}{\lambda_{s}}\left(1-F_{1}\left(k_{s} a\right)\right) \\
b / a & =F_{1}\left(k_{s} a\right)
\end{aligned}\right.
$$

where $0<k_{s} a \leq \infty$ is a parameter.

The optimal parameters are valid as long as the optimal shell is thinner than the shell corresponding to the first resonance. Since the optimal shell thickness found from (18) is always less than the resonance thickness, it is valid in the entire range of parameters $k a, \varepsilon_{\mathrm{r}}$. On the other hand, the validity of (14) is conditional. For $\varepsilon_{\mathrm{r}}=1$, the optimal parameters found using (14) can safely be used in the entire region where an antenna is regarded as electrically small $(k a<0.5)$, and actually up to $k a \approx 2$. In this range of $k a$, the shell thickness, at which the resonance occurs, exceeds the optimal shell thickness. For larger electrical sizes $k a$ and permittivity $\varepsilon_{\mathrm{r}}$, the applicability of (14) depends on $b / a$.

In the limit $b / a \rightarrow 1$, the optimal parameters (14) are valid irrespective of $k a$ and $\varepsilon_{\mathrm{r}}$. Indeed, as $k_{s} b \rightarrow k_{s} a$, the electrical size $k_{s} a$ becomes much larger than 1 (see Fig.3a). Consequently, the fields in the shell are locally plane waves, implying that the shell thickness for the internal resonance is $\lambda_{s} / 2$, whereas the optimal thickness found using (14) is approximately $0.2877 \lambda_{s}$.

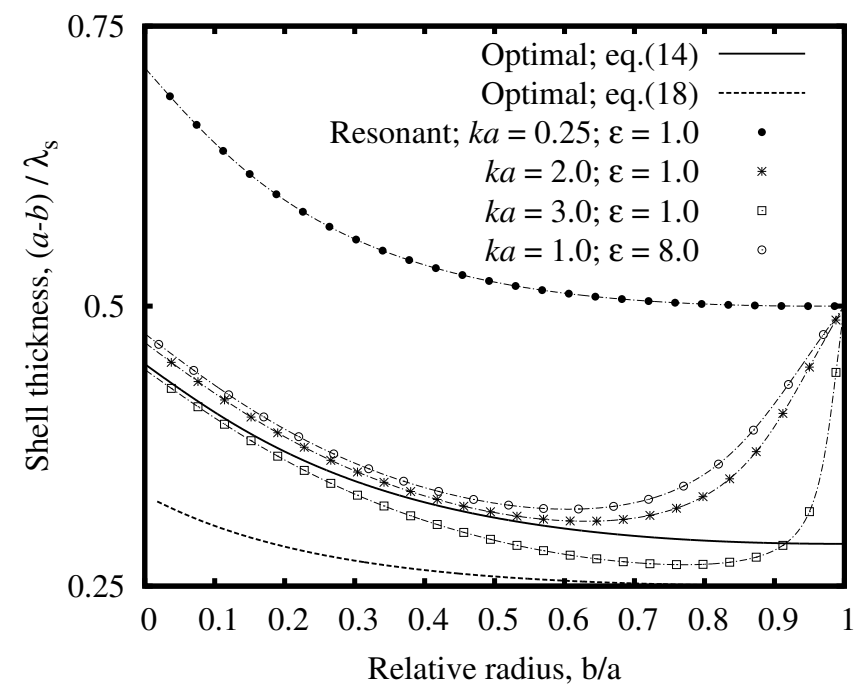

Fig. 5. Optimal shell thickness and shell thickness for the first resonance.

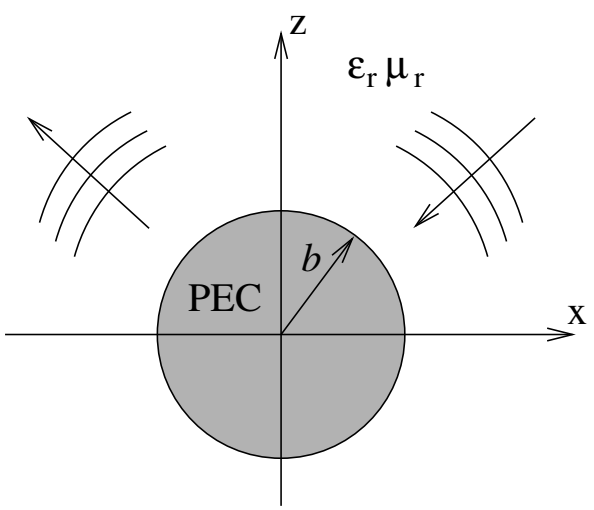

Fig. 6. Scattering of a spherical wave by a PEC sphere immersed in a medium with parameters $\varepsilon_{\mathrm{r}}, \mu_{\mathrm{r}}, k_{s}=\sqrt{\varepsilon_{\mathrm{r}} \mu_{\mathrm{r}}} k, \lambda_{s}=2 \pi / k_{s}$.

\section{B. A Standing Wave Model}

Optimal parameters can also be determined approximately by considering the material coating as a spherical waveguide with a PEC sphere of radius $b$ centered at the origin (Fig. 6). The PEC sphere is illuminated by an inward-propagating $\mathrm{TE}_{10}$ spherical wave, and, due to the spherical symmetry, the reflected outward-propagating wave is also a $\mathrm{TE}_{10}$ spherical wave. Since a spherical standing wave is formed, there is a radius $a>b$, at which the wave impedance is infinite. Placed at this radii an impressed electric current density (3) will radiate minimally inward, and, thus, the internal stored energy and the radiation $Q$ will be minimized. It can be shown that the radius $a$ of infinite impedance is given by the following equation

$$
\arg \left\{h_{1}^{(2)}\left(k_{s} a\right)\right\}=\arg \left\{h_{1}^{(2)}\left(k_{s} b\right)\right\}+\frac{\pi}{2} .
$$

For a vanishingly small PEC sphere $\left(k_{s} b \ll 1\right)$, the solution can be found analytically as

$$
k_{s} a=\chi_{p}
$$

where $\chi_{p}$ is the p-th root of the spherical Neumann function of first order $y_{1}(x)$. For finite $k_{s} b$, the equation (23) is solved 


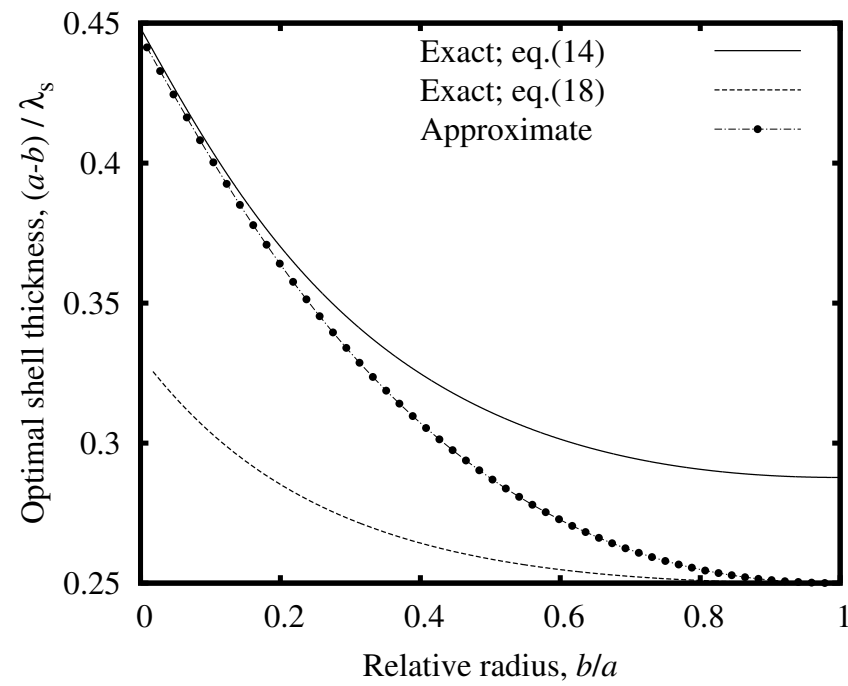

Fig. 7. Exact and approximate optimal thickness of the shell.

numerically and the result is shown in Fig. 7 along with the exact solutions found in Section III-A. It is observed, that for small $b / a$ the approximate solution is very close to the exact optimal parameters found from (14), whereas for larger $b / a$ the approximate solution approaches the exact optimal parameters found from (18). Thus, the standing wave model provides a reasonable approximation to the exact solution.

\section{CONCLUSION}

The radiation problem for an electric current density impressed on the surface of a material-coated PEC sphere and radiating the $\mathrm{TE}_{10}$ spherical mode is solved analytically. Closed-form expressions for electric and magnetic internal and external stored energies as well as for the radiation quality factor $Q$ are derived. From those, the optimal parameters of the antenna, which ensure the radiation $Q$ closest to the Chu lower bound, are determined.

It is shown that a PEC sphere introduced into a solid magnetic core shifts the core permeability, at which the first $\mathrm{TE}_{10}$-mode resonance occurs, towards higher values. Consequently, the optimal permeability also becomes higher, and this results in lower internal stored magnetic energy and lower $Q$. For instance, the optimum permeability of a solid magnetic core with $k a=0.5$ is $\mu_{\mathrm{r}}=31.72$, which corresponds to $Q=1.09 Q_{\mathrm{Chu}}$, whereas the optimum permeability of the same core with a PEC sphere of radius $b=0.9 a$ inside is $\mu_{\mathrm{r}}=1313.51$, and thus $Q=1.01 Q_{\mathrm{Chu}}$.

Furthermore, it is shown that the negative effect of the dielectric permittivity can be compensated by decreasing the thickness of the magnetodielectric coating. For an infinitely thin coating, the optimal permeability becomes infinite, and the $Q$ reaches the Chu lower bound, irrespective of the permittivity and antenna electrical size $k a$.

Thus, the realistic lower bound on the radiation $Q$ for spherical magnetic dipole antennas is indeed the Chu lower bound.

\section{ACKNOWLEDGMENT}

The authors thank Dr. Arthur Yaghjian for numerous fruitful discussions on the physical limits of electrically small antennas facilitating the work of this paper.

\section{REFERENCES}

[1] L. J. Chu, "Physical limitations of omni-directional antennas," J. Appl. Phys., vol. 19, no. 12, pp. 1163-1175, 1948.

[2] R. Collin and S. Rothschild, "Evaluation of antenna Q," IEEE Trans. Antennas Propagat., vol. 12, no. 1, pp. 23-27, Jan. 1964.

[3] J. S. McLean, "A re-examination of the fundamental limits on the radiation Q of electrically small antennas," IEEE Trans. Antennas Propagat., vol. 44, no. 5, pp. 672-676, May 1996.

[4] H. A. Wheeler, "The spherical coil as an inductor, shield, or antenna," Proc. IRE, vol. 46, no. 9, pp. 1595-1602, 1958.

[5] H. L. Thal, "New radiation Q limits for spherical wire antennas," IEEE Trans. Antennas Propagat., vol. 54, no. 10, pp. 2757-2763, Oct. 2006.

[6] R. Hansen and R. Collin, "A new Chu formula for Q," IEEE Antennas Propagat. Mag., vol. 51, no. 5, pp. 38-41, 2009.

[7] S. R. Best, "The radiation properties of electrically small folded spherical helix antennas," IEEE Trans. Antennas Propagat., vol. 52, no. 4, pp. 953-960, Apr. 2004.

[8] — , "Low Q electrically small linear and elliptical polarized spherical dipole antennas," IEEE Trans. Antennas Propagat., vol. 53, no. 3, pp. 1047-1053, Mar. 2005.

[9] - "A low Q electrically small magnetic (TE mode) dipole," IEEE Antennas Wireless Propagat. Lett., vol. 8, pp. 572-575, 2009.

[10] H. R. Stuart, H. R. Stuart, and C. Tran, "Small spherical antennas using arrays of electromagnetically coupled planar elements," IEEE Antennas and Wireless Propagat. Lett., vol. 6, pp. 7-10, 2007.

[11] O. S. Kim, "Low-Q electrically small spherical magnetic dipole antennas," IEEE Trans. Antennas Propagat., vol. 58, no. 7, pp. 2210-2217, July 2010.

[12] _ "Novel electrically small spherical electric dipole antenna," in Proc. of International Workshop on Antenna Technology (iWAT2010), Lisbon, Portugal, 2010.

[13] O. S. Kim, O. Breinbjerg, and A. D. Yaghjian, "Electrically small magnetic dipole antennas with quality factors approaching the Chu lower bound," IEEE Trans. Antennas Propagat., vol. 58, no. 6, pp. 1898-1906, June 2010.

[14] O. S. Kim and O. Breinbjerg, "Lower bound for the radiation Q of electrically small magnetic dipole antennas with solid magnetodielectric core," accepted for publication in IEEE Trans. Antennas Propagat., 2010.

[15] A. D. Yaghjian and S. R. Best, "Impedance, bandwidth, and Q of antennas," IEEE Trans. Antennas Propagat., vol. 53, no. 4, pp. 1298 1324, Apr. 2005

[16] A. Yaghjian, "Internal energy, Q-energy, Poynting's theorem, and the stress dyadic in dispersive material," IEEE Trans. Antennas Propagat., vol. 55, no. 61, pp. 1495-1505, 2007.

[17] A. D. Yaghjian and H. R. Stuart, "Lower bounds on Q for dipole antennas in an arbitrary volume," in Proc. IEEE Antennas Propag. Soc. Int. Symp., Toronto, Canada, July 11-17 2010, pp. 1-4.

[18] _ - "Lower bounds on the Q of electrically small dipole antennas," IEEE Trans. Antennas Propagat., vol. 58, no. 10, pp. 3114-3121, 2010.

[19] H. R. Stuart and A. D. Yaghjian, "Using high permeability shells to improve the $\mathrm{Q}$ of electrically small electric-dipole antennas," Proc. IEEE Ant. Propagat. Soc. Int. Sym., July 11-17 2010.

[20] — "Approaching the lower bounds on Q for electrically small electric-dipole antennas using high permeability shells," scheduled for publication in IEEE Trans. Antennas Propagat., vol. 58, no. 12, December 2010.

[21] O. S. Kim and O. Breinbjerg, "Decreasing the radiation quality factor of magnetic dipole antennas by a magnetic-coated metal core," in Proc. 20th International Conference on Applied Electromagnetics and Communications (ICECom 2010), Dubrovnik, Croatia, 20-23 September 2010.

[22] J. S. McLean, H. Foltz, and R. Sutton, "Broadband, electrically-small spherical-wire and generalized loop antennas exploiting inhomogeneous magnetic cores," in Proc. 20th International Conference on Applied Electromagnetics and Communications (ICECom 2010), Dubrovnik, Croatia, 20-23 September 2010.

[23] J. E. Hansen, Spherical Near-Field Antenna Measurements. London, U.K.: Peter Peregrinus, 1988. 
This is the author's version of an article that has been published in IEEE Transactions on Antennas and Propagation. Changes were made to this version by the publisher prior to publication. The final version of record is available at http://dx.doi.org/10.1109/TAP.2011.2158965

[24] R. L. Fante, "Quality factor of general ideal antennas," IEEE Trans. Antennas Propagat., vol. 17, no. 2, pp. 151-155, Mar. 1969.

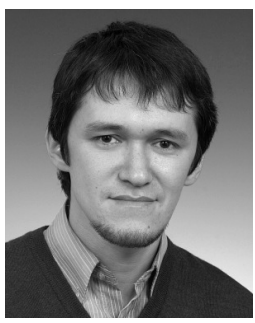

Oleksiy S. Kim received the M.S. and Ph.D. degrees from the National Technical University of Ukraine, Kiev, in 1996 and 2000, respectively, both in electrical engineering. In 2000, he joined the Antenna and Electromagnetics Group at the Technical University of Denmark (DTU). He is currently an associate professor with the Department of Electrical Engineering, Electromagnetic Systems, DTU.

His research interests include computational electromagnetics, metamaterials, electrically small antennas, photonic bandgap and plasmonic structures.

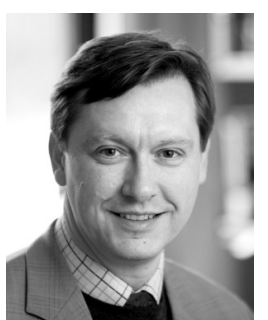

Olav Breinbjerg (M'87) was born in Silkeborg, Denmark on July 16, 1961. He received the M.Sc. and Ph.D. degrees in electrical engineering from the Technical University of Denmark (DTU) in 1987 and 1992, respectively. Since 1991 he has been on the faculty of the Department of Electrical Engineering (formerly Ørsted.DTU, Department of Electromagnetic Systems, and Electromagnetics Institute) where he is now Full Professor and Head of the Electromagnetic Systems Group including the DTU-ESA Spherical Near-Field Antenna Test Facility. Olav Breinbjerg was a Visiting Scientist at Rome Laboratory, Hanscom Air Force Base, Massachusetts, USA in the fall of 1988 and a Fulbrigt Research Scholar at the University of Texas at Austin, Texas, USA in the spring of 1995. Olav Breinbjerg's research is generally in applied electromagnetics - and particularly in antennas, antenna measurements, computational techniques and scattering - for applications in wireless communication and sensing technologies. At present, his interests focus on meta-materials, antenna miniaturization, and spherical near-field antenna measurements. He is the author or co-author of more than 40 journal papers, 100 conference papers, and 70 technical reports, and he has been, or is, the main supervisor of 10 $\mathrm{Ph}$.D.projects. Olav Breinbjerg has taught several B.Sc. and M.Sc. courses in the area of applied electromagnetic field theory on topics such as fundamental electromagnetics, analytical and computational electromagnetics, antennas, and antenna measurements at DTU, where he has also supervised more than 70 special courses and 30 M.Sc. final projects. Furthermore, he has given short courses at other European universities. He is currently the coordinating teacher at DTU for the 3rd semester course 31400 Electromagnetics, and the 79th semester courses 31428 Advanced Electromagnetics, 31430 Antennas, and 31435 Antenna Measurements in Radio Anechoic Chambers. Olav Breinbjerg received a US Fulbright Research Award in 1995. Also, he received the 2001 AEG Elektron Foundation's Award in recognition of his research in applied electromagnetics. Furthermore, he received the 2003 DTU Student Union's Teacher of the Year Award for his course on electromagnetics. 